\title{
Light driven structuring of glasses
}

\author{
B. P. Antonyuk, A.Z. Obidin*, and K.E. Lapshin* \\ Institute of Spectroscopy, Russian Academy of Science, 142190 Troitsk, Moscow Region, \\ Russia. E-mail: Antonyuk@isan.troitsk.ru*Physics Instrumentation Center, \\ General Physics Institute, Russian Academy of Science, \\ 142190 Troitsk, Moscow Region, Russia.
}

(Dated: August 4, 2021)

\begin{abstract}
Theoretical and experimental evidence of light driven structuring of glasses is presented. We show that light overcomes Coulomb repulsion and effective electron-electron interaction in glasses under strong light pumping becomes attractive. As the result homogenious distribution of trapped electrons gets unstable and macroscopic electron bunches are grown. At different conditions ordered structures with period $2 \mu m \pm 0.2 \mu m$ determined by internal properties of the material are formed These structures were observed in ablation: surface profile after laser treatment reveals ordered pattern corresponding to the light induced electron domains.
\end{abstract}




\section{INTRODUCTION}

Direct and obvious method of light driven structuring is the using of a special light field configuration. Generation of the light intensity grating and corresponding grating of excitations are widely discussed. More interesting and physically rich is structuring due to light driven self-organization. This ordering belongs to the class of self-organization phenomena in open dissipative systems and it is presented here. The typical examples of this class are Benard convection [1], Belousov-Zhabotinsky reactions [2] and Turing instability [3]. All self-organization phenomena are driven by some external flow through the system: heat in Benard convection, chemical reagents in Belousov-Zhabotinsky reactions, etc. Optical analogue of Turing instability was presented in [4]. In the latter case light amplitude has been modulated in time and space, which was crucial for the organization to occur. Our investigations(see [5], 6], 7] and references therein) have shown that steady light flow through a system can provide ordering either. This light is a driving force which bunches the electrons in the case in hand. An electron in disordered media (we shall call them "glasses") moves in random potential forming its energy spectrum. It consists of the extended electron and hole bands separated by the "gap" filled by the local states. We shall discuss here the case when photon energy is less than the gap in glass and light generates transitions between local states only. Transitions between different traps are of a spacial interest. They are responsible for the light induced current and the structuring of the matter discussed. The electron transfer from an initial trap $i$ to the final trap $f$ may be treated as generation of electron and hole at sites $f$ and $i$ respectively. Strong Coulomb electron-hole interaction is changed considerably with the shift of the distance between traps $f$ and $i$ therefore strong electron-phonon coupling takes place. Light absorption in the case of strong electron-phonon interaction is well studied. The rate $w_{f i}$ of the electron transition between sites $i$ and $f$ is given by the formula [8]

$$
w_{f i}=I \sigma_{0} \cos ^{2} \theta_{f i} \exp \left(-\frac{\left(\hbar \omega-\epsilon_{f i}-A\right)^{2}}{\Delta^{2}}-\kappa_{f i} R_{f i}\right) \quad\left(\epsilon_{f i}>0\right),
$$

where $I$ is photon flux, $\hbar \omega$ is photon energy, $\epsilon_{f i}=\epsilon_{f}-\epsilon_{i}$ is the energy of the electron excitation (difference between electron energies $\epsilon_{f}$ in trap $f$ and $\epsilon_{i}$ in trap $i$ ); $\mathbf{R}_{\mathbf{f i}}=\mathbf{R}_{\mathbf{f}}-\mathbf{R}_{\mathbf{i}}$; $R_{f i}=\left|\mathbf{R}_{\mathbf{f}}\right|$, where vectors $\mathbf{R}_{f}$ and $\mathbf{R}_{i}$ points positions of the traps; $\theta_{f i}$ is the angle between $\mathbf{R}_{f i}$ and the light polarization vector, $\sigma_{0} \approx 10^{-18} \mathrm{~cm}^{2}, \Delta \approx 10^{-1} \div 10^{-2} \mathrm{eV}, A \approx 10^{-1} \div 10^{-2} \mathrm{eV}$ is the Stokes shift. The light driven electron transitions in hand are accompanied by phonon 
generation. Phonons are excluded from the formula (11) therefore exact energy conservation law $\hbar \omega=\epsilon_{f i}+\hbar \Omega(\hbar \Omega$ is phonon energy) looks like conservation with the accuracy $\Delta \approx \hbar \Omega$ : according to (11) an electron may be transfered to different levels in the range of the band width $\Delta$. Absorption band (11) as a function of $\epsilon_{f i}$ gains maximum (resonance) at the energy $M=\hbar \omega-A$. We shall call the excited states with the $\epsilon_{f i}<M$ as low energy excitations (deep levels) and excited states with the $\epsilon_{f i}>M$ as high energy ones.

\section{LIGHT DRIVEN BUNCHING OF ELECTRONS}

As a first step we shall examine light driven kinetics of a single electron in external electric field $\mathbf{E}$. It is nontrivial: as we shall see electron is shifted by light predominantly in opposite to the electric force $\mathbf{F}=\mathbf{e E}$ direction. The idea of such behavior is following. If vector of electron transfer is $\mathbf{R}=\mathbf{R}_{f}-\mathbf{R}_{i}$ then dipole moment generated is $\mathbf{d}=e \mathbf{R}$ ( $e<0$ is electron charge). Field contribution to the energy of the excitation is $\delta \epsilon_{f i}=-(\mathbf{d E})=-e(\mathbf{R E}) \equiv$ $-(\mathbf{R F})$. If the electron is transported in opposite to the electric force direction $((\mathbf{R F})<\mathbf{0})$ the energy of the electron excitation $\delta \epsilon_{f i}$ becomes larger; low energy excitation becomes closer to resonance $M$ and therefore probability of its generation increases. This means that electron transfer to the deep levels goes predominantly in opposite to the electric force direction. Electron transitions to high levels go mainly in the direction of the force but these levels have less life time in comparison with deep levels therefore contribution of the deep levels prevails. In the case of two electrons according to the above idea light would push each electron in the direction of the other electron. Let the second electron is embedded at positions $j$ then Coulomb interaction shifts all energy levels for the first electron and, hence, shifting the excitation energies by $e^{2} / R_{f j}-e^{2} / R_{i j}$ for the transition $i \longrightarrow f$ of the first electron. Transitions which reduces electron-electron distance give positive contribution to the Coulomb energy and shift deep excitations towards the resonance $M$. That is why low energy transitions of an electron are directed preferably towards the other electron and effective electron-electron interaction becomes attractive under strong pumping. Due to shorter life time high energy excitations play minor role in comparison with deep levels and therefore contribution of low energy excitations dominates. At high pumping these transitions dominate also over ordinary mobility which is quite low and independent on laser power. Effective attraction between electrons makes their homogeneous distribution 
unstable and electron bunches are formed.

Phonon assistance designates two peculiar features of our system: first, it is dissipative (not Hamiltonian); secondly, phonons brake phase relations for the electron wave functions therefore nondiagonal elements of the density matrix vanish and only probabilities of the site populations appear in consideration and they give full and correct description of the system. If the energy difference $\epsilon_{f i}<0$ (electron loses energy), there are two channels to transit: first, it can undergo light-induced transition with the rate being described in a way analogous to (10) with the only change $A$ to $-A([\underline{8}])$; second, it may relax to a lower energy state with the rate $\gamma_{f i}=\gamma_{0} \exp \left(-\kappa_{f i} R_{f i}\right)$. So, for the electron transition process with the energy loss we have:

$$
w_{f i}=I \sigma_{0} \cos ^{2} \theta_{f j} \exp \left(-\frac{\left(\hbar \omega-\epsilon_{i f}+A\right)^{2}}{\Delta^{2}}-\kappa_{f i} R_{f i}\right)+\gamma_{0} \exp \left(-\kappa_{f i} R_{f i}\right) \quad\left(\epsilon_{f i}<0\right)
$$

The factor $\gamma_{0}$ is of the order of the inverse lifetime for excited electron states in an atom, so that $\gamma_{0} \approx 10^{8} \mathrm{~s}^{-1}$. Light driven kinetics is governed by dimensionless pumping constant $\mu=\frac{I \sigma_{0}}{\gamma_{0}}$. Wave function of trapped electron outside the trap $i$ is $\Psi_{i}(\mathbf{R}) \propto e^{-\kappa_{i}\left|\mathbf{R}-\mathbf{R}_{i}\right|}$, where $\kappa_{i}=\sqrt{\frac{2 m}{\hbar^{2}}\left(V-\epsilon_{i}\right)}, m$ is the electron mass, $V$ is spacing of bottom of trapped levels from the bottom of the extended states. Normal scale for this parameter is $\kappa_{0}=\sqrt{\frac{2 m}{\hbar^{2}} V} \approx 10^{7} \mathrm{~cm}^{-1}$. Wave function of deep levels vanishes rapidly outside the trap while shallow levels have long tails of wave functions. Their overlap is determined by the slowest function, therefore $\kappa_{f i}=\kappa_{0} * \min \left\{\sqrt{1-\frac{\epsilon_{f}}{V}}, \sqrt{1-\frac{\epsilon_{i}}{V}}\right\}([\underline{5}])$.

In the frame of the (11), (2) master rules we studied light driven motion of two electrons and calculated their density-density correlation function

$$
K(R)=\frac{1}{T R^{d-1}} \int_{0}^{T} n\left(\mathbf{R}_{i}\right) n\left(\mathbf{R}_{j}\right) d t
$$

where $\left.R=\left|\mathbf{R}_{i}-\mathbf{R}_{j}\right|\right)$ and $n$ are the occupation numbers of the corresponding traps, $t$ is time. We normalize the correlation function by the factor $R^{d-1}$, where $d$ is dimensionality of the system. This normalization avails to see the interaction effect: if the electrons were not interacting, one would have $K(R) \equiv$ const for the noncorrelated case. The typical $K(R)$ for three dimensions is shown in Fig. [1]

The bar chart displays the probability of different electron-electron ranges. Electron traps were randomly distributed in space and single electron level $\epsilon_{i}^{0}$ in each trap $i$ is also random according to some density of states. One electron in position $j$ shifts each initial level $i$ of 


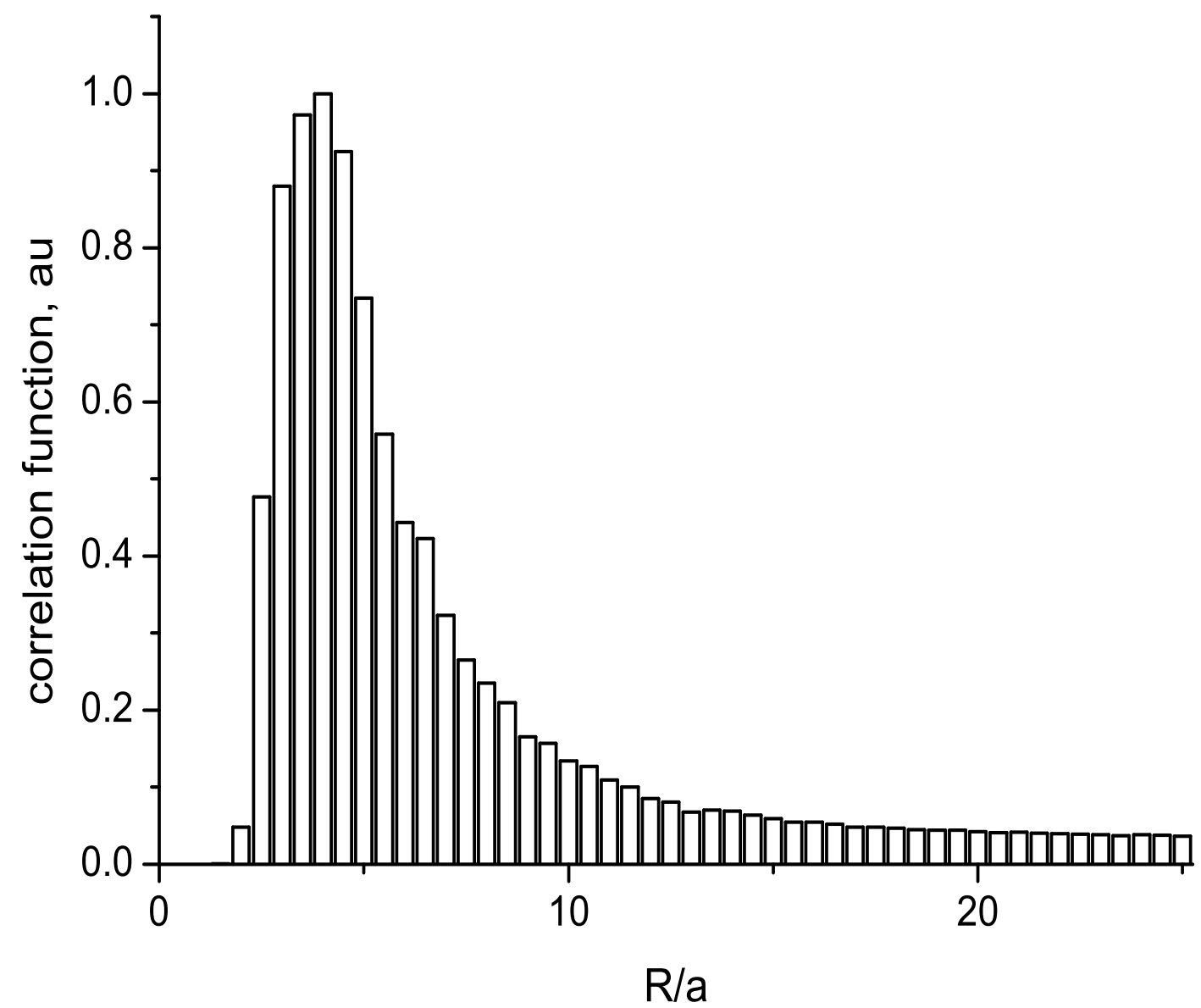

FIG. 1: Electron-electron correlation function revealing the bielectron state. The parameters are: $\hbar \omega=0.2 \mathrm{eV}, \mu=100, V=0.3 \mathrm{eV}$, electron density is constant within the range $0 \div V$.

the second electron to the energy of Coulomb interaction (and vice versa)

$$
\epsilon_{i}=\epsilon_{i}^{0}+\frac{e^{2}}{R_{i j}}
$$

The parameters used for calculation are: average trap distance $a=5 \AA, \kappa_{0}=1 / a, \hbar \omega=$ $0.2 \mathrm{eV}$, impurity band width $V=0.3 \mathrm{eV}, \Delta=0.1 \mathrm{eV}, A=0.1 \mathrm{eV}$. The bound state of two electrons is formed at pumping constant $\mu=\frac{I \sigma_{0}}{\gamma_{0}}>1$. Pumping constant $\mu=1$ corresponds to laser power $10^{7} \mathrm{~W} / \mathrm{cm}^{2}$. We found that at high pumping in wide region of parameters the correlation function has such a maximum. Despite the Coulomb repulsion two electrons prefer to stay at some finite distance. Analogous behavior has been observed for $d=2$ and 
$d=1$. Increase of average distance $a$ between the traps (decrease of Coulomb interaction) results in narrowing and vanishing of the peak in $K(R)$. At lower $\mu$ the peak in $K(R)$ vanishes also (see for the details [6]).

Electron motion reveals competition between ordinary mobility in the direction of the electric force $e \mathbf{E}$ given by relaxation term $\propto \gamma_{0}$ in (2) and light driven electron transfer in opposite direction. At long distance between the electrons the light induced transitions dominate and an electron is pushed to the other electron: light overcomes Coulomb repulsion (!). This motion is stopped at short distances where Coulomb repulsion dominates: electrons

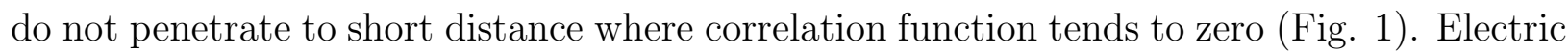
field at this distance is $E=e / R^{2}=10^{7} \mathrm{~V} / \mathrm{cm}$ and this is an estimation for the effective electric field caused by light induced electron transfer. This field is close to the damage field $3 * 10^{7} \mathrm{~V} / \mathrm{cm}$. As the result of the competition bound state of two electrons is formed with some preferable distance between particles. Effective attraction between electrons results in light driven electron bunching. We examined behavior of few electrons or even $10 \div 100$ electrons and found quite similar peculiarities. Energy shift of the initial levels for hopping electron is analogous to (44) but is given by all other electrons:

$$
\epsilon_{i}=\epsilon_{i}^{0}+\Sigma_{j} \frac{e^{2}}{R_{i j}}
$$

We studied temporal change of the average size of the electron cloud $R^{2}=$ $1 / 2 \Sigma_{i j} R_{i j}^{2}, R_{i j} \equiv\left|\mathbf{R}_{i}-\mathbf{R}_{j}\right|$ and Coulomb energy $1 / 2 \Sigma_{i j} e^{2} / R_{i j}$. The result is shown in Figs. 2, 3, electron cloud is pressed by light into compact bunch. We see again electron motion which is impossible in thermodynamic case: the system tends to the state with higher energy (Fig. 31). Bunch formation is accompanied by strong increase of the electric field at the boundary of the bunch. The formation is stopped by this field when it gains the value $\approx 10^{7} \mathrm{~V} / \mathrm{cm}$. It is convenient to introduce ground state and electron-hole presentation for the investigation of many body system in glass. Glass never attain full thermodynamic equilibrium but partial equilibrium is possible and one may consider that at ground state all electron levels bellow some energy $\epsilon_{F}$ are occupied and states above this level are empty. Energy shift of a level is determined in this case by the contribution of all electrons and holes available.

$$
\epsilon_{i}=\epsilon_{i}^{0}+\sum_{j}^{e} \frac{e^{2}}{R_{i j}}-\Sigma_{j}^{h} \frac{e^{2}}{R_{i j}}
$$




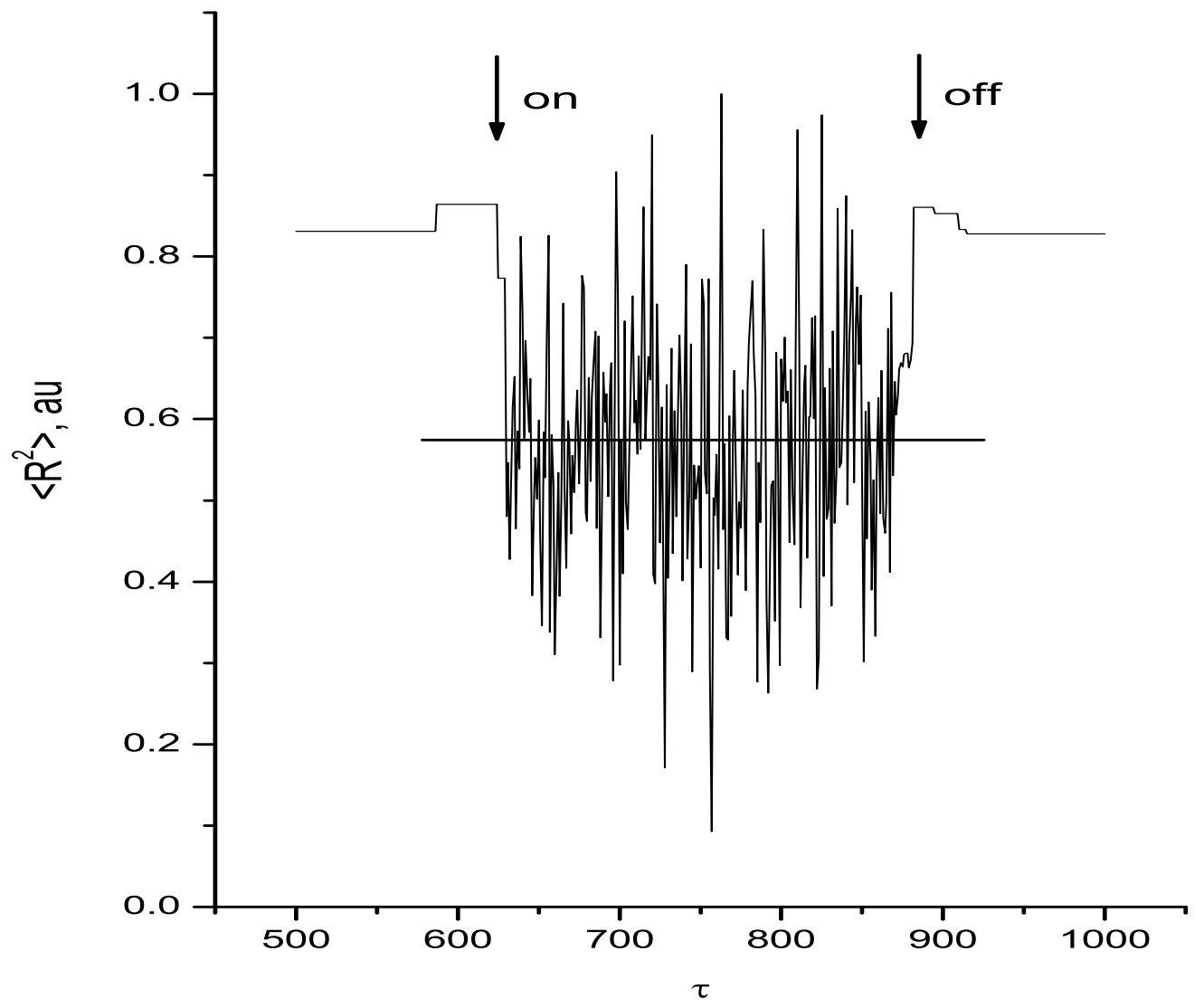

FIG. 2: Light driven bunching of the electrons. Squared size of the electron cloud $<R^{2}>$ is decreased under light pumping (parameters are $E_{0}=0, a=50 \AA, 1 / \kappa=50 \AA, \mu=100$, number of electrons $N=10$.).

Contribution to the energy of the first excited electron is given by its "own" hole only

$$
\epsilon_{i}=\epsilon_{i}^{0}-\frac{e^{2}}{R_{i j}}
$$

where $j$ is position of the hole. Calculation of light induced polarization have shown that it is directed in opposite to an external field direction (Fig. 4) and therefore amplifies this field.

The studied three dimensional many body system with long range Coulomb interaction reveals self-organization effect even at weak pumping $\mu \ll 1$. We examined behavior of 


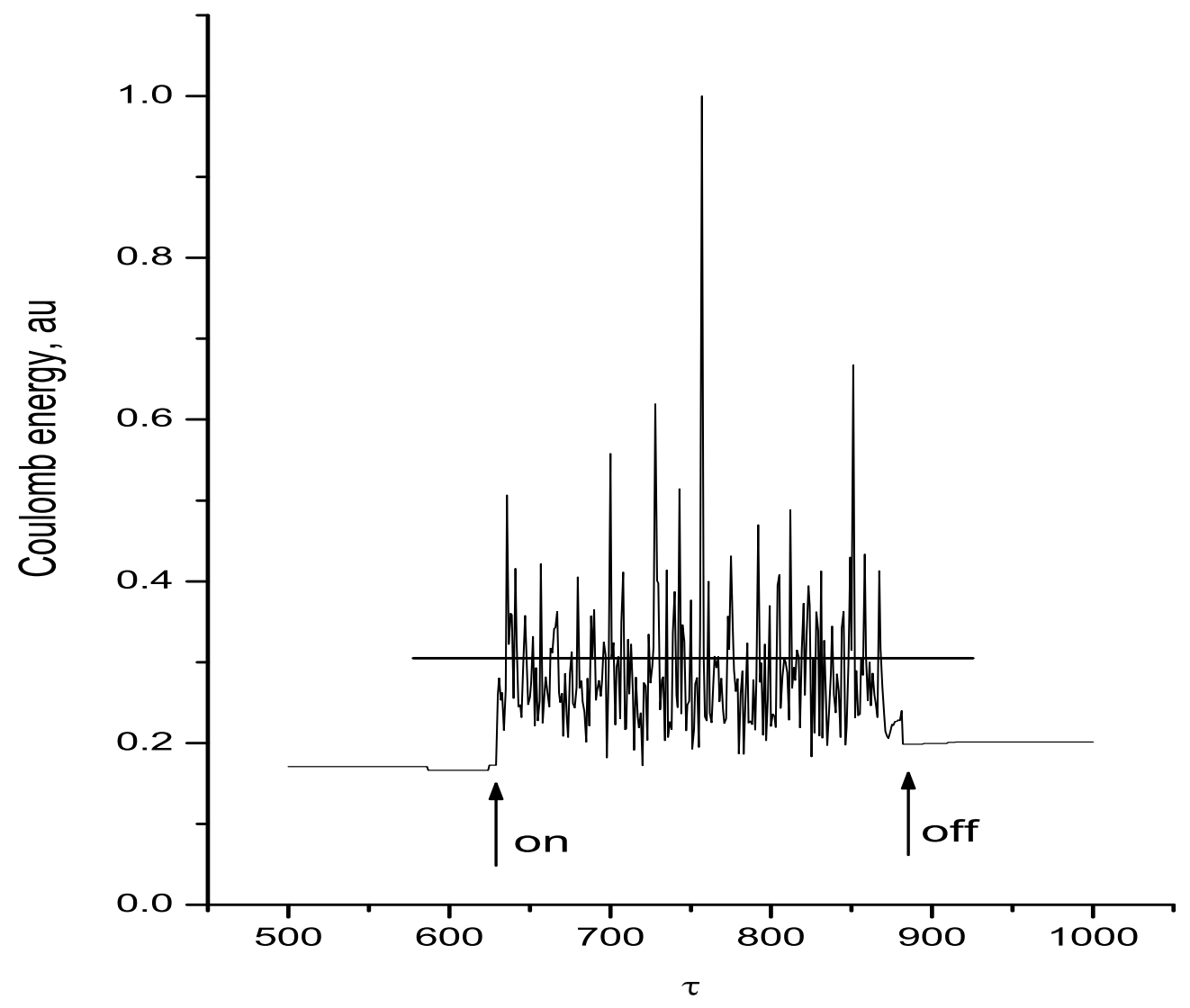

FIG. 3: Increase of electron Coulomb energy due to bunching under light pumping (parameters are $\left.E_{0}=0, a=50 \AA, 1 / \kappa=50 \AA, \mu=100, N=10\right)$.

many body system of different size for one-, two- and three-dimensional samples. In main features it is the same for all dimensions. The corresponding model is presented in details in [5], 6], 7]. Computer simulation of the electron kinetics in the finite sample under external electric field shows that in agreement with the above idea electrons are transported by light predominantly in the direction opposite to the electric force (electric current flows contra the voltage drop). This transfer is in contrast to the electron motion in a thermodynamic case when electrons move in the direction of the external force reducing in accordance with the Le Chatelet principle the external field (negative feedback). In our case glass reveals positive feedback in response to the electric field. As a result strong static polarization di- 


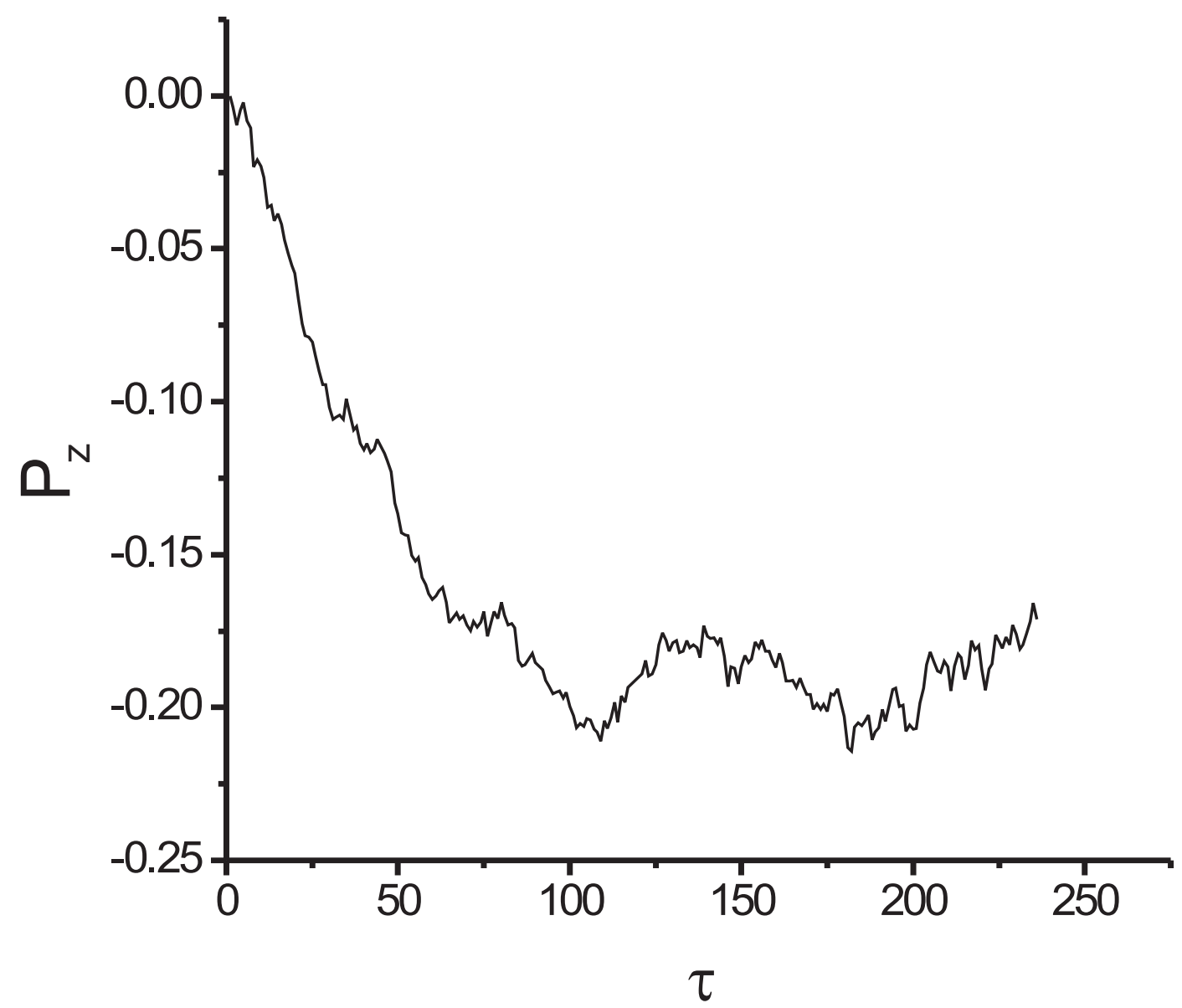

FIG. 4: Light induced polarization of the sample in $|e| / a^{2}$ units vs dimensionless time $\tau=\gamma_{0} t$ for parameters $a=150 \AA, \mu=0.001$, external field $E_{0} \equiv E e a / \Delta=0.225, E a^{2} / e=0.02, \Delta=0.01 e V$. rected in opposite to the external electric field is established. This polarization amplifies the initial field considerably. At the field of saturation light induced electron transitions are in the dynamical equilibrium with the ordinary electron mobility in opposite direction. Computer simulation of the light driven electron-hole kinetics have shown positive feedback in response to static electric field in wide region of parameters independently on the dimension of the system. This unusual response makes homogeneous electron distribution unstable: an electron moves towards another electron or towards a cluster of electrons and macroscopic bunches are formed. 


\section{EXPERIMENTAL}

We performed special experiment in order to observe directly the discussed bunching. Strong laser wave with photon energy $\hbar \omega \approx 7 \mathrm{eV}$ was used. Band gap in our glass $\epsilon_{g} \approx 8 \mathrm{eV}$ and therefore direct generation of free electrons and holes is impossible. However in the region of strong electric field due to Franz-Keldysh effect [9], 10] this process becomes effective. Energy discrepancy $\delta \epsilon$ between the glass gap and photon energy is gained from the static electric field in process of electron tunneling (the corresponding estimation see in 6]). So, domain boundaries due to strong electric field become a weak chain in glass bonds and light driven bond breaking (local fusion) reveals bunch structure of the self-organized state. High quality UV-grade fused silica samples (KU-1) with thicknesses of $1 \div 10 \mathrm{~mm}$ have been used in the experiment. Before the experiment the samples have been subjected to ultrasonic cleaning in acetone and ethanol followed by washing in de-ionized water. Two beams from the unstable resonator of the $A r F$ excimer laser (193nm, CL-7000, PIC GPI) were focused on the rear side of a sample by the lens made of $M g F_{2}$. We changed the angle between beams and therefore produced grating with different periods at the output surface of the glass. ArF excimer laser generated 20ns FWHM pulses with energies up to $350 \mathrm{~mJ}$ and pulse repetition rate up to $100 \mathrm{~Hz}(\mu>>1)$. Ablation has been performed in ambient air as well as in a hermetic chamber that was purged by nitrogen or evacuated. Silica surface morphology and etched structures depth have been analyzed with an optical microscope with further recording with Cohu-4812 CCD camera. Photograph of the surface after the light treatment is presented in Fig. 5.

One can see farrows (grating) corresponding to the light intensity grating with the period $\approx 10 \mu m$ and self-organized structure of each of them with the period $2 \mu m \pm 0.2 \mu m$ corresponding to the discussed here self-organized electron bunches. This size has nothing in common with the light field scale (wavelength, beam size, coherent length etc.), it is determined by internal properties of the glass what is characteristic feature of self-organized system and coincides exactly with the period of the bubble structure found in single wave experiment shown in Fig. 6] 6]. We changed coherent length of the light waves, beam sizes, beam power and found that surface profiles (Figs. 15, 6) remain the same: all experiments reveals the same spatial period $2 \mu m \pm 0.2 \mu m$ what is typical for the self-organized systems. The material sputtering (ablation) occurs on the rear side of a fused silica plate with no 


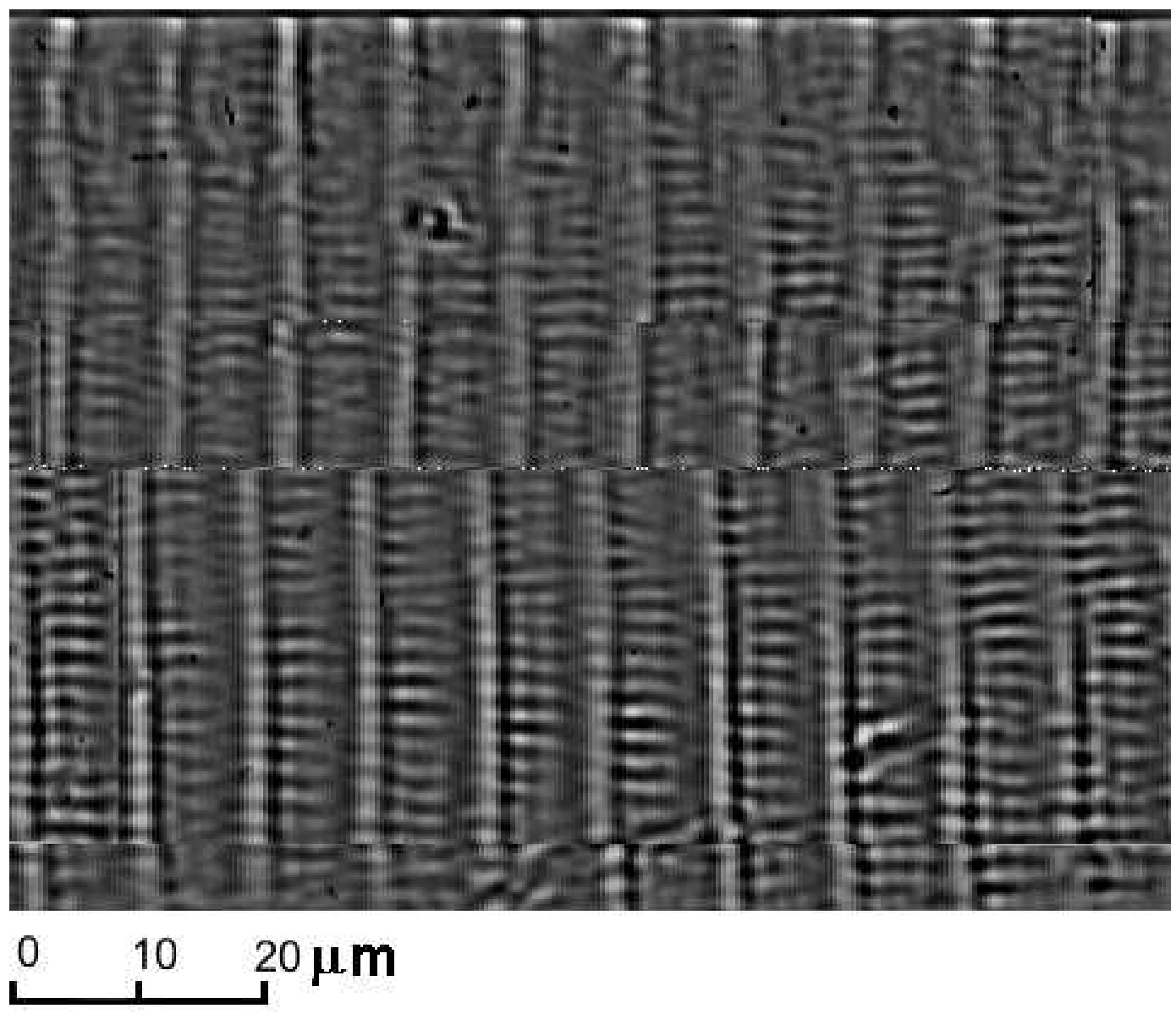

FIG. 5: Laser treated surfaces after ablation in two wave experiment: forced grating is structured by self-organized system of domains with the period of the bubble structure found in single wave experiment shown in Fig. 6. Spatial coherence length exceeds $10 \mathrm{~mm}$.

stimulation of the process (such as plasma generation in the vicinity of a sample or specific UV absorbing medium [11]). Analogous behavior was observed in 12] where surface structuring took place at the $\mathrm{SiO}_{2}-\mathrm{Si}$ interface and was explained in another way as interface property.

\section{CONCLUSIONS}

Our theoretical and experimental study of light driven electron kinetics in silica glass have shown that homogeneous distribution of the trapped electrons becomes unstable under light pumping and macroscopic electron (hole) bunches are formed. In wide region of the external parameters light pushes electrons into micron size coagulums which we observed in ablation 


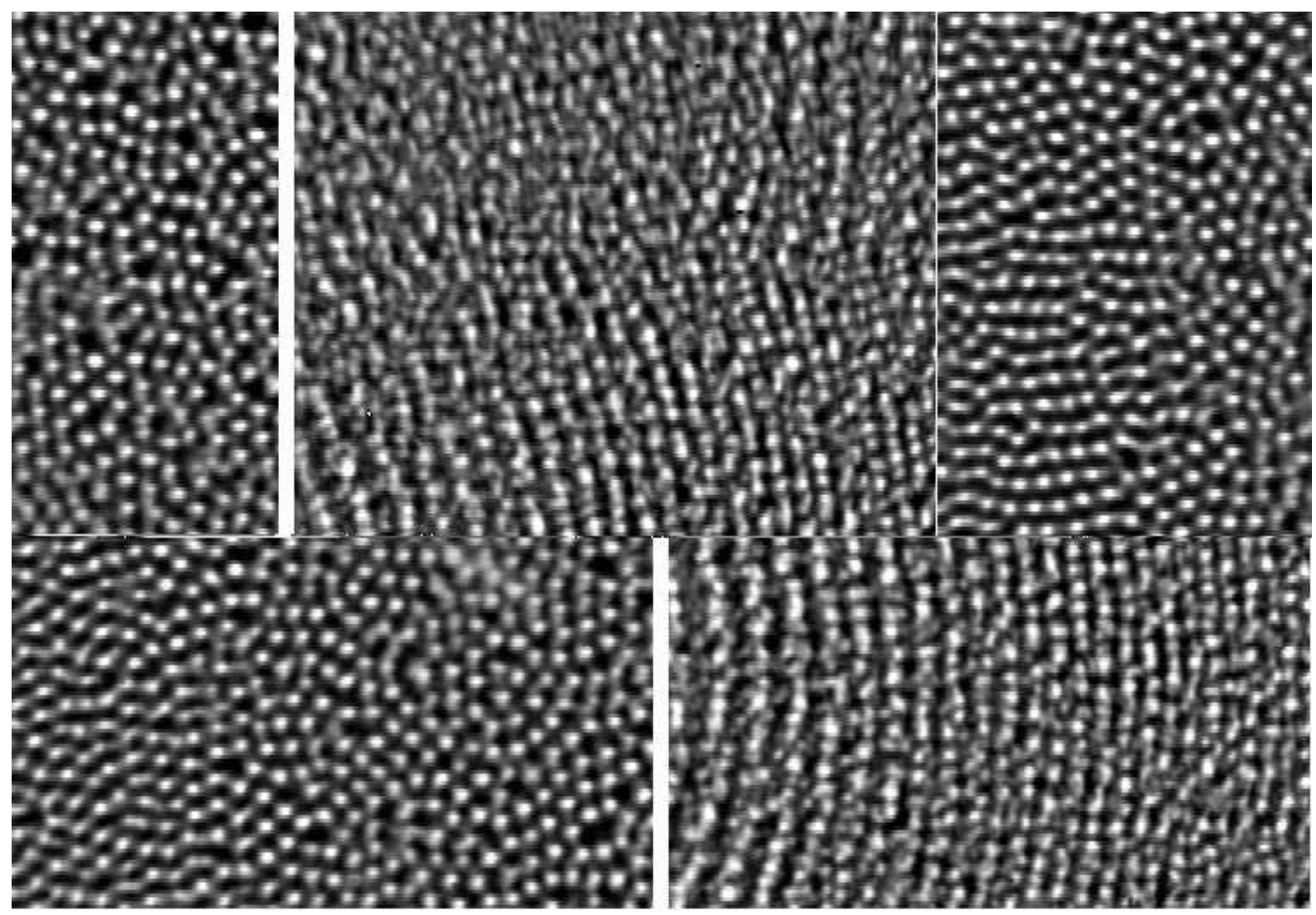

$\begin{array}{llll}0 & 10 & 20 \mu \mathrm{m} & \text { (a) }\end{array}$

(b)

FIG. 6: Laser treated surfaces in single wave experiment [6]: a) surface obtained after 300 pulses (laser fluence is $\approx 3 J / \mathrm{cm}^{2}$ ) of incoherent beam (spatial coherence length is less then $10 \mu m ; \mathrm{b}$ ) surface obtained by highly coherent radiation treatment with spatial coherence length $>6 \mathrm{~mm}$ in all directions. Structures with the same spatial period $2 \mu m \pm 0.2 \mu m$ are formed at different conditions a), b), and Fig. 5 .

process. Under different conditions structures with the same spatial period $2 \mu m \pm 0.2 \mu m$ are formed. This new length is determined by the internal properties of the material and is idependent on light properties (beam size, beam power, coherent length etc.) It was found that UV beam $\lambda=193 \mathrm{~nm}$ provides bunching and subsequent local bond breaking effectively but wave $\lambda=248 \mathrm{~nm}$ can not drive this process. Deeper electrons with short tails of electron wave functions are involved in this case $\left(\kappa_{f i}\right.$ is increased $)$ and probabilities of the transitions are less. In addition the energy discrepancy $\delta \epsilon$ is increased therefore probability of Franz-Keldysh tunneling becomes negligable. We observed also red luminescence of the glass sample under UV pumping recently reported in number of papers (see [1]] and cited 
in [5] references). Ordering under light pumping discussed here may be the basement of the so called "compaction effect" (see [13] and references therein) being the serious problem in lithography. Ordered by light glass state certainly is more compact.

\section{ACKNOWLEDGEMENTS}

This work was performed under auspices of Russian Academy of Sciences in the frame of the program "Optical spectroscopy and frequency standards" and Russian Foundation for the Basic Research under Grant 04 -02-16394.

[1] H. Benard, Rev. Gen. Sci. Pures Appl. 121261 (1900).

[2] A.N. Zaikin, A.M. Zhabotinsky, Nature 225535 (1970).

[3] A.M. Turing, Phil. Trans. R. Soc. London B 23737 (1952).

[4] F.T. Arecchi, Physica D 86297 (1995).

[5] B.P. Antonyuk and V.B. Antonyuk, Physics-Uspekhi 4453 (2001).

[6] B.P. Antonyuk, V.B. Antonyuk, A.Z. Obidin, S.K. Vartapetov and S.K. Kurzanov Opt. Comm 230151 (2004).

[7] Boris P. Antonyuk, "Light Driven Self-Organization", Nova Science Publishers, Inc., NY (2003).

[8] R. Kubo and Y. Toyazawa, Progr. Theor. Phys. 13160 (1955).

[9] W.Z. Franz, Naturforsh. Bd. 13A s. 484 (1958).

[10] L.V. Keldysh, JETP 341138 (1958).

[11] K. Sugioka, J. Zhang, K. Midorikawa, Laser Machining Method and Laser Machining Apparatus, Patent No.: US 6180915 B1, (2001).

[12] J.J. Yu, J.V.Zhang, I.W. Boyd and Y.F. Lu, Appl. Phys. A 7235 (2001).

[13] R. Schenker and W. Oldham, J. Vac. Sci. Technol B 143709 (1996). 